\title{
OP-0122 Triglycerides/glucose index, a cost effective, screening marker of insulin resistance among children and adolescents
}

E. Dikaiakou1, E. Vlachopapadopoulou'1, I. Panagiotopoulos', M. Kafetzi², E. Kaloumenou1,

A. Fotinou ${ }^{2}$, S. Michalacos ${ }^{1}$

1. Dept. of Endocrinology-Growth and Development 2. Biochemistry Dept.-Hormones Laboratory

Children's Hospital P. \& A. Kyriakou, Athens, Greece

\section{BACKGROUND}

Childhood obesity is one of the most serious public health challenges of the 21 st century. Obesity-related insulin resistance is present in obese children and adolescents and is one of the leading causes of metabolic abnormalities.

AIMS:

To investigate the association between the triglyceride / glucose index (TyG Index) with TG/HDL and with the HOMAIR and MATSUDA insulin resistance indices

$>$ To estimate whether it could be used as a predictor of insulin resistance in overweight and obese children and adolescents.

\section{METHODS}

\&34 children ( $40 \%$ boys) , overweight or obese.

Mean age $10.8 \pm 2.3$

* After an overnight fast glucose, lipid profile and insulin levels were measured.

* HOMA-IR was calculated. TyG index was calculated as

In [triglycerides $(\mathrm{mg} / \mathrm{dl}) \times$ fasting glucose $(\mathrm{mg} / \mathrm{dl}) / 2$ ].

* In a subset of 95 children oral glucose tolerance test

(OGTT) was performed and MATSUDA index was calculated.

\section{RESULTS}

$\checkmark$ TyG and TG/HDL were significantly and positively correlated $(p<0.001)$ with HOMA-IR with correlation coefficients equal to 0.54 and 0.55 , respectively.

$\checkmark$ TyG and TG/HDL were significantly and negatively correlated $(p<0.001)$ with Matsuda index with correlation coefficients equal to -0.55 and -0.34 , respectively.

$\checkmark$ TyG was positively correlated with TG/HDL $(r=0.81, p<0.001)$

$\checkmark$ Mean levels of TyG were higher in children with insulin resistance $(8.31 \pm 0.47$ vs. $7.87 \pm 0.43, p<0.001)$.

$\checkmark$ TG/HDL mean levels were greater in those with insulin resistance $(1.53 \pm 0.89$ vs. $2.60 \pm 2.34, p=0.011)$.

The area under the curve was $0.75(95 \% \mathrm{Cl}: 0.63-0.87, \mathrm{p}<0.05)$.

$\checkmark$ The optimal cutoff values for predicting insulin resistance as evidenced by HOMA> 3.0 was equal to $\geq 7.96$ with a sensitivity of $78.1 \%$ and a specificity of $64.1 \%$.

$\checkmark$ The optimal cut off values of TyG index for predicting insulin resistance as evidenced by MATSUDA $<2.5$ was equal to $\geq 7.92$ with a sensitivity of $85.2 \%$ and a specificity of $60.7 \%$.

$\checkmark$ The area under the curve was $0.76(95 \% \mathrm{Cl}: 0.65-0.87, \mathrm{p}<0.05)$.

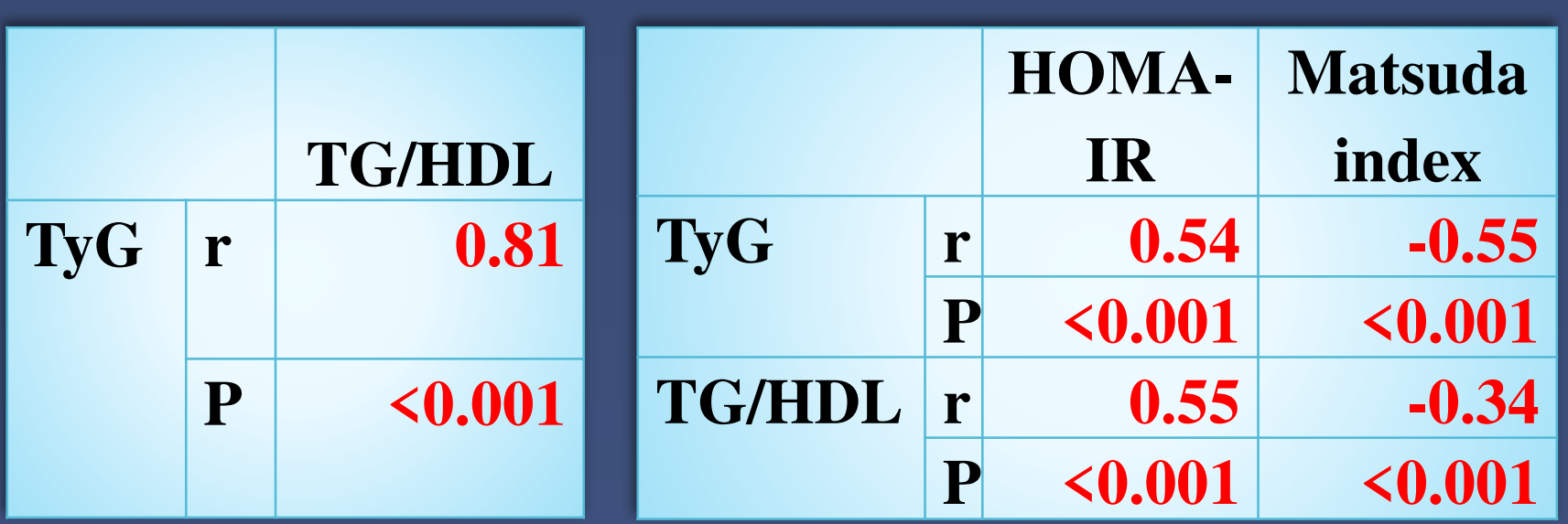

\begin{tabular}{|c|c|c|c|c|c|}
\hline & \multicolumn{4}{|c|}{ (HOMA-IR>3) } & \multirow{3}{*}{$\begin{array}{c}\text { P } \\
\text { Student' } \\
\text { s t-test }\end{array}$} \\
\hline & \multicolumn{2}{|c|}{ No } & \multicolumn{2}{|c|}{ Yes } & \\
\hline & Mean & SD & Mean & SD & \\
\hline TyG & 7.86 & 0.41 & 8.31 & 0.52 & $<0.001$ \\
\hline TG/HDL & 1.44 & 0.77 & 2.70 & 2.19 & 0.002 \\
\hline
\end{tabular}

\begin{tabular}{|l|r|r|r|r|r|}
\hline \multirow{2}{*}{} & \multicolumn{4}{|c|}{ Insulin resistant $<2.5$} & \multirow{2}{*}{ p Student's } \\
\cline { 2 - 6 } & \multicolumn{2}{|c|}{ No } & \multicolumn{2}{c|}{ Yes } & p \\
\cline { 2 - 6 } & Mean & SD & Mean & SD & t-test \\
\hline TyG & 7.87 & $\mathbf{0 . 4 3}$ & $\mathbf{8 . 3 1}$ & 0.47 & $<0.001$ \\
\hline TG/HDL & 1.53 & $\mathbf{0 . 8 9}$ & 2.60 & 2.34 & 0.011 \\
\hline
\end{tabular}
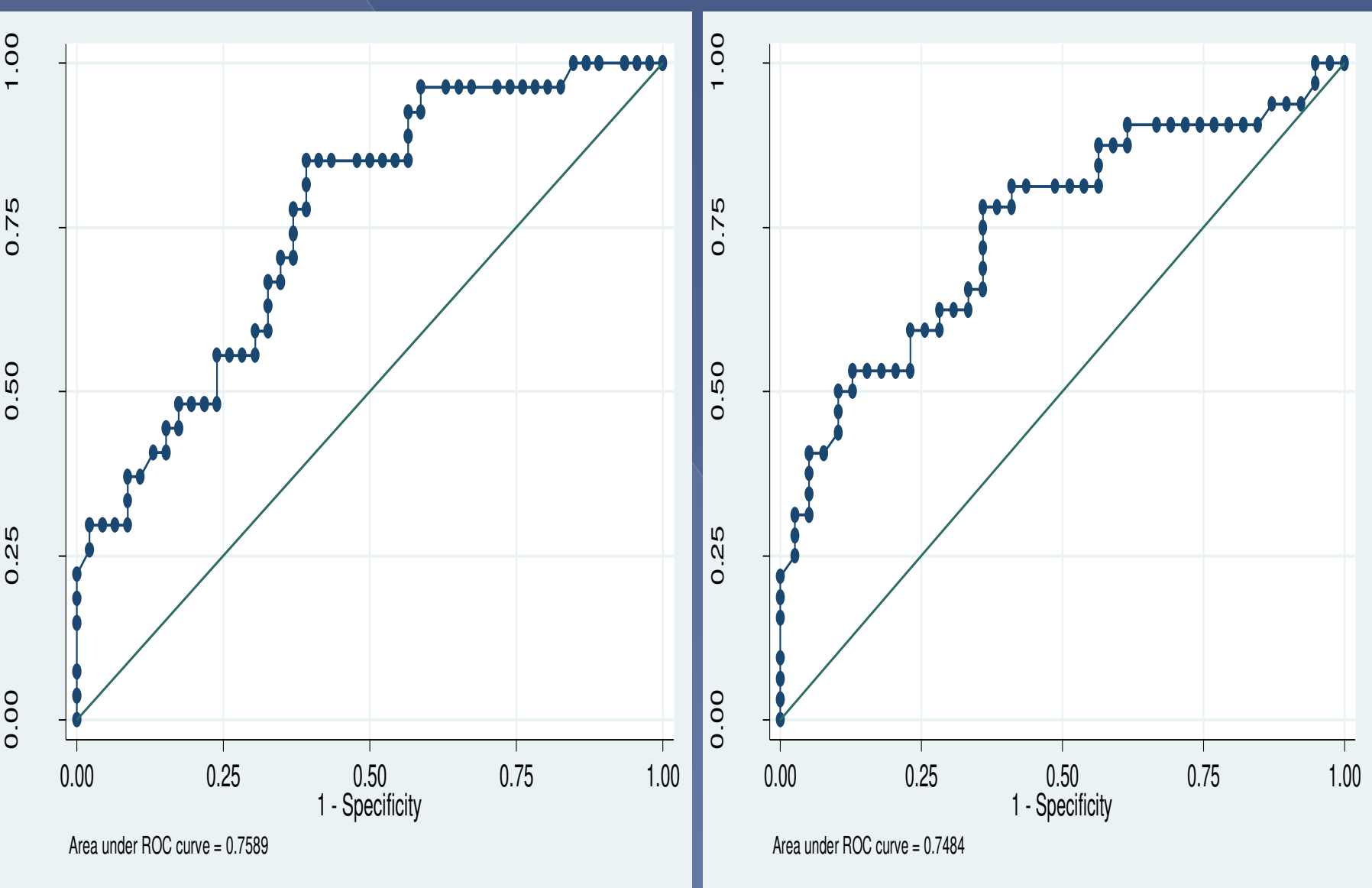

\section{DISCUSSION}

The TyG index is a simple, cost-effective surrogate marker of insulin resistance among overweight and obese children and adolescents. It can be used as screening for insulin resistance among overweight and obese children and adolescents. It could be a useful indication in clinical practice to highlight children who will need OGTT. 